\title{
Multiple autoimmune antibody limbic encephalitis: a case in a pregnant woman
}

\author{
Meha Goyal ${ }^{1}$, Kasey L. Gildersleeve ${ }^{2}$, Stuart L. Tomko' ${ }^{1}$, Joseph S. Kass ${ }^{2,3}$ \\ ${ }^{1}$ Department of Neurology, Baylor College of Medicine, Houston, TX 77030, USA. \\ ${ }^{2}$ Department of Neurology, The University of Texas Health Science Center, Houston, TX 77030, USA. \\ ${ }^{3}$ Department of Neurology, Ben Taub General Hospital, Houston, TX 77030, USA.
}

\section{A B S T R A C T}

Autoimmune limbic encephalitis is most commonly associated with antibodies against the N-methyl-D-aspartate receptor (NMDAR), among other neuronal cell surface receptors. Here, a case of a pregnant female with limbic encephalitis in the presence of multiple additional autoimmune antibodies is described. The patient was a 36-year-old female who presented with 4 days of confusion, hallucinations, hypersexuality, disinhibition, and pressured speech. The patient's work-up detected the presence of anti-NMDAR antibodies, anti-glutamic acid decarboxylase antibodies, and a yet uncharacterized neuronal autoantibody. The patient was also found to be pregnant. No evidence of ovarian or other pelvic malignancy was discovered. Symptomatic control was achieved with plasma exchange.

Key words: Autoimmune, encephalitis, glutamic acid decarboxylase, limbic, N-methyl-D-aspartate

\section{INTRODUCTION}

Autoimmune limbic encephalitis is most commonly associated with antibodies against the $\mathrm{N}$-methyl-D-aspartate receptor (NMDAR). ${ }^{[1]}$ Several other autoimmune antibodies have been implicated in autoimmune limbic encephalitis, but the existence of several of these antibodies in one patient has rarely been described. Autoimmune limbic encephalitis manifests as sub-acute onset of irritability, short-term memory loss, depression, sleep disturbances, hallucinations, seizures, and confusion. ${ }^{[1,2]}$ Anti-NMDAR encephalitis is the most common form, whereas other antibodies described with this syndrome are directed at amino-3-hydroxy-5-methyl-4-isoxazolepropionic acid receptor (AMPAR), gamma-aminobutyric acid beta receptor (GABA-BR), leucine-rich glioma inactivated (LGI1) gene, and contactin-associated protein $2{ }^{[3]}$ Anti-glutamic acid decarboxylase (GAD) antibodies, although most commonly associated with autoimmune-mediated Stiff-Person syndrome, have also

\begin{tabular}{|l|l|}
\hline \multicolumn{2}{|c|}{ Access this article online } \\
\hline Quick Response Code: & \\
\hline & Website: \\
\hline & Www.nnjournal.net \\
\cline { 2 - 3 } & \\
\hline
\end{tabular}

been described in association with late-onset cerebellar ataxia, epilepsy, palatal tremor, and limbic encephalitis. ${ }^{[4-6]}$ However, the clinical significance of these antibodies in the context of encephalitis has been questioned given the small number of cases reported as well as the frequent co-presence of antibodies against cell surface antigens (e.g. GABA-BR, AMPAR, and NMDAR). ${ }^{[4-6]}$ Cases of anti-GAD receptor encephalitis described in the literature generally do not follow the clinical pattern of typical limbic encephalitis. Patients with anti-GAD receptor encephalitis present with prominent memory loss, medically refractory seizures, and language difficulty. ${ }^{[2]}$ In addition, patients with autoimmune encephalitis can also demonstrate seropositivity for other antibodies such as anti-nuclear antibody and anti-thyroperoxidase (TPO). Immunomodulatory treatment remains the gold standard of therapy, with first-line treatments consisting of corticosteroids, intravenous immunoglobulin (IVIG), or plasma exchange (PLEX); second-line treatment includes cyclophosphamide and rituximab. ${ }^{[1]}$ Here, we describe a case of a pregnant female found to have anti-NMDAR encephalitis with the presence of multiple autoimmune antibodies.

\section{CASE REPORT}

A 36-year-old female presented with 4 days of confusion, hallucinations, hypersexuality, disinhibition, and pressured speech. Initial evaluation showed a thyroid

Corresponding Author: Dr. Meha Goyal, Baylor College of Medicine, 7575 Kirby Drive 2303, Houston, TX 77030, USA.

E-mail: mgoyal@bcm.edu 
stimulating hormone level of $<0.01$, free thyroid hormone level (T4) of 3.53 positive anti-TPO and thyroid stimulating immunoglobulin, suggesting hyperthyroidism-induced psychosis. Methimazole treatment resulted in a euthyroid state, but no symptomatic improvement. On day 4, brain magnetic resonance image (MRI) showed no acute intracranial process. Due to concerns that the MRI was performed too early to detect changes, it was repeated 1-month after presentation. This second scan was also unremarkable. The patient's electroencephalogram showed no epileptiform activity during sleep or wakefulness.

Cerebrospinal fluid (CSF) analysis on day 8 yielded lymphocytic pleocytosis (white blood cell (WBC) count of 44 with $95 \%$ lymphocytes) with negative bacterial cultures, fungal cultures, and viral polymerase chain reactions. A 3 days course of IV steroids for inflammatory encephalitis yielded no symptomatic improvement. On day 17, repeat CSF studies showed persistent pleocytosis (WBC count of 44 with $3 \%$ lymphocytes). A second trial of IV steroids for 7 days followed by oral steroid taper again failed to achieve symptomatic improvement. CSF and serum samples were sent to two laboratories for further testing, specifically for neuronal cell surface antigens and synaptic proteins such as NMDAR, AMPAR, GABA-BR, and autoantigens. One laboratory, the Josep Dalmau laboratory, uses recombinant technology, while the other, the ARUP laboratory at the University of Utah, uses immunofluorescence to identify antibodies. On day 24, the initial CSF samples returned positive for anti-NMDAR antibody (titer 1:5). The patient was thus diagnosed with anti-NMDAR encephalitis; no anti-NMDAR antibodies were detected in the serum. Scans for malignancy with whole body computed tomography images uncovered a thickened uterine endometrium but no evidence of ovarian masses; this finding was confirmed by transvaginal ultrasound. The patient repeatedly refused a pelvic MRI.

For the management of her neuropsychiatric symptoms, the patient received olanzapine, valproate, and haloperidol for psychosis control and mood stabilization. However, as she developed significant Parkinsonian features (hypertonicity, mask-like facies, tremor), haloperidol was discontinued, and benztropine and diphenhydramine were added with subsequent resolution of Parkinsonism.

As the patient failed repeated steroid treatments, alternate treatments were considered. While IVIG or PLEX would be first-line treatments for anti-NMDAR encephalitis, there was concern that the patient would not tolerate an indwelling central line given her agitation, mania, and psychosis. Thus, Rituximab therapy was recommended. Prior to initiation of Rituximab, a urine pregnancy test (UPT) was repeated on day 36 and found to be positive (admission UPT had been negative). Subsequent plasma human chorionic gonadotropin test was positive and transvaginal ultrasound confirmed a viable fetus.

On discovery of a viable fetus, all teratogenic medications were discontinued, folate supplements were started, and the plan for rituximab therapy was abandoned. At this time, the patient's psychosis and agitation were more controlled. She seemed to exhibit more restraint due to knowledge of her pregnancy and her care for the child's health. Thus, she tolerated the central line well. After extensive discussions with the patient, family, and consulting services, the patient underwent seven cycles of PLEX over 2 weeks (days: 44-58), with a resolution of psychosis and bizarre behavior. During this time, additional CSF and serum analyses came back positive for anti-GAD antibody (titers unavailable), and a yet unidentified neuronal autoantibody found in CSF only; no other antibodies were identified (e.g. AMPAR, GABA-BR). Clinically, the patient did not demonstrate sequelae of Stiff-Person syndrome. The patient was largely asymptomatic by day 58 and discharged home on prenatal vitamins, folate, and levothyroxine. At 4-month follow-up, the patient was symptom-free and found to have subclinical hyperthyroidism.

\section{DISCUSSION}

Three cases of anti-NMDAR encephalitis in pregnant women have been described in the literature, ${ }^{[7]}$ but to the best of our knowledge, this is the first with multiple autoimmune antibodies present. Our patient demonstrated two distinct antibodies, anti-NMDAR and anti-GAD, as well as another neuronal autoantibody that has not yet been characterized. Both the anti-NMDAR and anti-GAD antibodies have been described in association with autoimmune limbic encephalitis, though our patient's titer of anti-NMDAR antibody (1:5) was significantly lower than previously described cases $(>1: 80) \cdot{ }^{[7]}$ It is unclear whether one of the antibodies was the dominant cause of encephalitis or if the three worked synergistically. Our patient was atypical for anti-NMDAR encephalitis in that she had less severe symptoms and never progressed to autonomic instability or central hypoventilation. Although she likely experienced a few seizures, she never experienced the typical movement disorders of anti-NMDAR encephalitis. Her disease also manifested much earlier in the pregnancy (5 weeks of gestation) compared with previous gravid patients who presented at 8,14 , or 17 weeks of gestation. ${ }^{[7]}$ She responded well to PLEX, with significant symptomatic improvement by the fifth cycle. She was also atypical for anti-GAD 
encephalitis; never developing cerebellar ataxia, epilepsy, or palatal tremor.

The role of autoimmune disease in pregnancy is brought to question in this case. Not only did our patient develop symptoms of anti-NMDAR encephalitis much earlier in pregnancy than in previously described cases, but she also began having symptoms coincidentally close to conception. Based on ultrasound dates, the patient likely conceived within days of presenting to our facility. Her symptoms of autoimmune encephalitis also began 4 days prior to presentation. In this case, there exists a temporal relationship between the time of conception and development of the patient's disease process. Although, there is evidence showing that patients with autoimmune diseases-such as multiple sclerosis and rheumatic disease-may have fewer symptoms during pregnancy, anti-NMDAR encephalitis in our patient could potentially have been precipitated by pregnancy. Further studies will be needed to determine if this is simply a correlation or if there is true causation.

This case also illustrates the need for an extensive search for the etiology of a patient's neuropsychiatric symptoms. Prior to the discovery of the described neuronal autoantibodies, a patient presenting with Hashimoto's thyroiditis and neuropsychiatric symptoms would have been diagnosed with Hashimoto's encephalopathy. ${ }^{[5]}$ However, even with anti-TPO antibodies, a more exhaustive search for other antibodies is required in order to gain full appreciation of the patient's disease. Furthermore, Hashimoto's encephalopathy is responsive to steroids. Steroid-refractory disease should lead clinicians to investigate other etiologies of a patient's symptoms. Autoimmune limbic encephalitis should be considered in patients with psychosis refractory to medications and no identifiable cause.

\section{REFERENCES}

1. Titulaer MJ, McCracken L, Gabilondo I, Armangué T, Glaser C, Iizuka T, Honig LS, Benseler SM, Kawachi I, Martinez-Hernandez E, Aguilar E, Gresa-Arribas N, Ryan-Florance N, Torrents A, Saiz A, Rosenfeld MR, Balice-Gordon R, Graus F, Dalmau J. Treatment and prognostic factors for long-term outcome in patients with anti-NMDA receptor encephalitis: an observational cohort study. Lancet Neurol 2013;12:157-65.

2. Sharma A, Dubey D, Sawhney A, Janga K. GAD65 Positive autoimmune limbic encephalitis: a case report and review of literature. J Clin Med Res 2012;4:424-8.

3. Rosenfeld MR, Dalmau JO. Paraneoplastic disorders of the CNS and autoimmune synaptic encephalitis. Continuum (Minneap Minn) 2012;18:366-83.

4. Graus F, Saiz A, Dalmau J. Antibodies and neuronal autoimmune disorders of the CNS. J Neurol 2010;257:509-17.

5. Matà S, Muscas GC, Naldi I, Rosati E, Paladini S, Cruciatti B, Bisulli F, Paganini M, Mazzi G, Sorbi S, Tinuper P. Non-paraneoplastic limbic encephalitis associated with anti-glutamic acid decarboxylase antibodies. J Neuroimmunol 2008;199:155-9.

6. Saiz A, Blanco Y, Sabater L, González F, Bataller L, Casamitjana R, Ramio-Torrenta L, Graus F. Spectrum of neurological syndromes associated with glutamic acid decarboxylase antibodies: diagnostic clues for this association. Brain 2008;131: 2553-63.

7. Kumar MA, Jain A, Dechant VE, Saito T, Rafael T, Aizawa H, Dysart KC, Katayama T, Ito Y, Araki N, Abe T, Balice-Gordon R, Dalmau J. Anti-N-methyl-D-aspartate receptor encephalitis during pregnancy. Arch Neurol 2010;67:884-7.

Cite this article as: Goyal M, Gildersleeve KL, Tomko SL, Kass JS. Multiple autoimmune antibody limbic encephalitis: a case in a pregnant woman. Neuroimmunol Neuroinflammation 2015;2(1):46-8.

Source of Support: Nil. Conflict of Interest: No.

Received: 19-08-2014; Accepted: 30-09-2014 